\title{
DEVELOPMENT AND FORMULATION OF Azospirillum lipoferum AND Pseudomonas fluorescens AS EFFECTIVE BIOLOGICAL AGENTS FOR ENHANCED AGRO-PRODUCTIVITY
}

\author{
DESENVOLVIMENTO E FORMULAÇÃO DE Azospirillum lipoferum E Pseudomonas \\ fluorescens ENQUANTO AGENTES BIOLÓGICOS EFICAZES PARA UMA AGRO- \\ PRODUTIVIDADE MELHORADA
}

\author{
Saravanakumar TAMILSELVI ${ }^{1}$; Asmita DUTTA ${ }^{1}$; M SINDHU ${ }^{1}$, G subbayan MURUGESAN ${ }^{1}$; \\ Rengaraju BALAKRISHNARAJA ${ }^{1}$ \\ 1. Departament of Biotecnology, Bannari Amman Instituto de Tecnologia,Sathyamangalam, TN, Índia. balakrishnarajar@bitsathy.ac.in
}

\begin{abstract}
Biofertilizer is a group of beneficial microorganisms used for improving the productivity of soil by fixing atmospheric nitrogen or by solubilizing soil phosphorus. They also stimulate plant growth through synthesis of growth promoting substances. In this present study, Azospirillum lipoferum is grown in Nitrogen free Bromothymol blue (Nfb) medium and Pseudomonas fluorescens in King's B medium. Bioprocess condition was optimized for both of the culture and found that Pseudomonas fluorescens has shown highest growth at $30^{\circ} \mathrm{C}$ in $\mathrm{pH} 8$ after 72 hours of incubation where as Azospirillum lipoferum showed highest cell concentration at $31^{\circ} \mathrm{C}$ in $\mathrm{pH} 7$, with incubation period of 72 hours. The optimized culture is mixed with different formulations of powder and liquid carrier such as Saw dust, Rice husk, Date seed powder, Matka khad, Jiwamrit and Beejamrit respectively. Shelf life study for 0, 30, 60, 90 and 120 days by cell counting and spread plate method showed that shelf life of the biofertilizer produced from Powder and liquid carriers had high amount of viable microbial population up to 120 days storage. Among biofertilizer based bio inoculants, Saw dust showed maximum population of $77 \times 10^{9} \mathrm{cfu} / \mathrm{ml}$ for Azospirillum lipoferum and $72 \times 10^{9} \mathrm{CFU} / \mathrm{ml}$ for Pseudomonas strain on $120^{\text {th }}$ day and the liquid carrier Matka khad showed $85 \times 10^{9} \mathrm{cfu} / \mathrm{ml}$ for Azospirillum lipoferum and $78 \times 10^{9} \mathrm{CFU} / \mathrm{ml}$ for Pseudomonas fluorescens.
\end{abstract} formulations.

KEYWORDS: Biofertilizers. Azospirillum lipoferum. Pseudomonas fluorescens. Powder carriers and liquid

\section{INTRODUCTION}

Biofertilizers are the bioinoculants of specific beneficial microorganisms that promote the growth of plant crops by converting the unavailable form of nutrients into available form. These biofertilizers also induce resistance in plants against pests, to improve soil fertility, to help plant growth by increasing the number and biological activity of desired microorganisms in the root surface (SIVASAKTHIVELAN et al., 2013). Azospirillum is a nitrogen fixing biofertilizer that colonizes in the root. Bacteria produces growth-promoting substances like indole acetic acid (IAA), gibberellins, pantothenic acid, thiamine and niacin and it also increases the rootlet density and root branching resulting in the increased uptake of mineral and water (VIJENDRAKUMAR et al., 2014). Azospirillum belongs to the family of Rhodospirillaceae and order Rhodospirillales associated with roots of monocots and can fix Nitrogen of $20-40 \mathrm{~kg} / \mathrm{ha}$, in addition to growth regulating substances. The Azospirillum form relationship with many plants mostly with those having the C4-dicarboxylic pathway of photosynthesis and Slack pathway, because they grow and fix nitrogen on salts of organic acids such as malic, aspartic acid (KAUSHAL et al.,2013). Azospirillum under stress conditions enhance plant growth by fixing atmospheric nitrogen. Production of growth promoting substances influence root development by increased uptake of nutrients from the land, and inhibiting pathogenic fungi and bacteria in the rhizosphere. (HOSSAIN ET al., 2015).

Pseudomonas fluorescence is a very common gram negative bacteria. It has antagonistic activity. It can produce some secondary metabolite or some antifungal compound such as fluorescent pigments, siderophores, hydrocyanic acid (HCN) and more important lytic enzymes. These lytic enzymes can degrade the chitin, $\beta$-1,3-glucan and protein components present on the fungal cell wall (RAMYASMRUTHI et al.,2012). It produces IAA and Promote enhancement of root length, shoot length, or number of lateral root (WAHYUDI et al., 2011). It is reported that Pseudomonas fluorescence is able to produce an antifungal metabolite called pyrrolnitrin, mainly used against Rhizoctonia Sp. and Fusarium Sp. (ANBUSELVI et al., 2010). It 
also has a potential of phosphate solubilisation, if it is placed on Pikovskaya media it forms a clear halo zone (FEKADU et al., 2013). So Pseudomonas acts as both biofertilizer and biopesticide.

A Bioinoculant can improve product stability, shelf life and also protect bacteria against different environmental conditions and provide initial food source. Application of PGPR either to increase crop health or to manage plant diseases depending on the development of bioformulations with suitable carriers that maintain the survival of bacteria for a considerable length of time (JAMBHULKAR et al., 2014).They can be applied for seed treatment, bio priming, seedling, foliar spray, fruit spray and sucker treatment (RITIKA et al., 2014). Therefore, liquid inoculants formulation with good field performance characteristics that uses low cost materials and are easily attainable by small producers who could overcome many problems associated with processing solid carriers (SIVASAKTHIVELAN et al., 2012).

In this study, Azospirillum lipoferum and Pseudomonas fluorescence were optimized for bioprocess conditions such as $\mathrm{pH}$, temperature, incubation period and effective formulation were made by using liquid and powder carriers that promote the growth of the bacteria for the usage as an effective biological agent.

\section{MATERIAL AND METHODS}

\section{Microorganisms}

Azospirillum lipoferum (Nitrogen Fixer) and Pseudomonas fluorescence were procured from the Bannari Amman Sugars-BIOLAB, Sathyamangalam. Azospirillum lipoferum was maintained on Nitrogen Free Bromothymol blue Malate (Nfb) (Deshwal et al., 2013).

\section{Optimization of the growth condition of bacterial strains}

The growth conditions such as $\mathrm{pH}$, temperature and incubation period of Azospirillum lipoferum and Pseudomonas fluorescence were optimized and the data were analyzed using MAT lab Version-8.1.0.604 (Prema et al., 2013).

\section{Microbial analysis of different carriers Formulations of Powder carriers}

Various organic materials and agricultural wastes such as Saw dust, Rice husk and Date seeds powder were used for the mass multiplication of Azospirillum and Pseudomonas using the methodology of Marjan et al., 2011.

\section{Formulation of Liquid Carriers}

Vedic krishi inputs such as Matkakhad, Beejamrit, Jiwamrit were the liquid carriers formulated using the methodology of Sanjay et al., 2012.

\section{Shelf life of bioinoculants}

The shelf lives of bioinoculants were checked for the different carriers'viz. powder and liquid carriers by spread plate technique. MATLAB Version-8.1.0.604 is used to analyse the shelf life of carriers.(Jorjani et al., 2011).

\section{Field study}

Field performance of each formulations both liquid and powder has checked using Shallot (small onion, collected from Sathyamangalam). Different morphological characteristics for onion has scrutinized by ANOVA Agres Stat Version 3.1. Least Significant Difference test (LSD) at $1 \%$ probability level was applied to compare the differences among treatment mean values.

\section{RESULTS AND DISCUSSION}

The culture of Azopirllum lipoferum and Pseudomonas fluorescens were maintained in Nitrogen free Bromothymol blue ( $\mathrm{Nfb}$ ) medium and King's B medium respectively

\section{Optimization of growth condition for Bacterial strains}

Azospirillum lipoferum maintained in $\mathrm{Nfb}$ medium showed maximum growth at $\mathrm{pH} 7$, in $31^{\circ} \mathrm{C}$ after 74 hours of incubation (Figure 1A, 1B, 1C).

Motiur et al., 2006 studied that the isolates of Azospirillum,MR-3, MR-4 and MR-8 showed maximum growth at $41^{\circ} \mathrm{C}$ and rest of the isolates showed maximum growth between $35^{\circ}$ and $37^{\circ} \mathrm{C}$. The isolates MR-1, MR-3, MR-4, MR-7, MR-8 and MR-13 showed optimum growth at $\mathrm{pH} 8.0$ and the isolates MR-6, MR-11, MR-14, MR-15 and MR-16 showed optimum growth at $\mathrm{pH}$ 7.0, A.amazonense (MR-5) showed optimal growth at $\mathrm{pH}$ 6.5.

The optimal incubation period, $\mathrm{pH}$, temperature for Pseudomonas fluorescens was found to be $8,30^{\circ} \mathrm{C}$ and 72 hours (Figure 2A, 2B, 2C) respectively.

Prema et al., (2013) optimised Pseudomonas culture for the maximum production of siderophore with optimal medium composition such as $0.5 \mu \mathrm{M}$ iron, $55 \mu \mathrm{M}$ glucose, $30^{\circ} \mathrm{C}, \mathrm{pH}$ 7.0andincubation time of $72 \mathrm{hrs}$. 


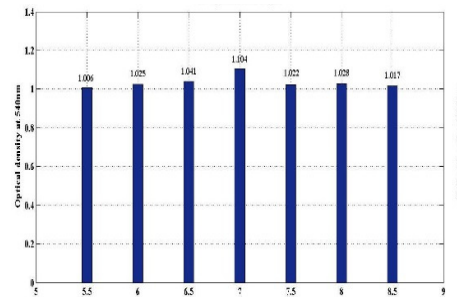

1A-pH (Azospirillum)

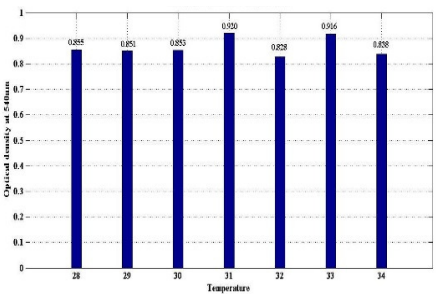

1B-Temperature (Azospirillum)

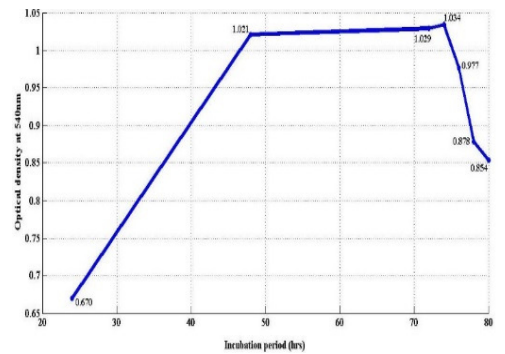

1C- Incubation period (Azospirillum)

Figure 1. Optimization of $\mathrm{pH}$, Temperature and Incubation period of Azospirillum lipoferum

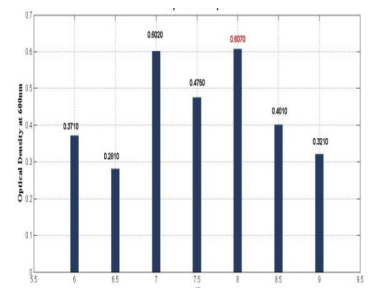

2A - pH (Pseudomonas)

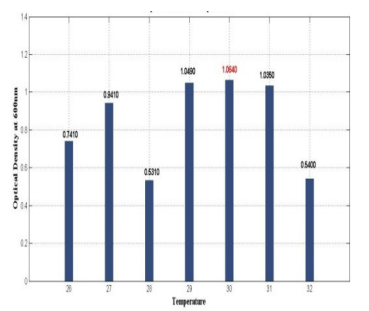

2B - Temperature (Pseudomonas)

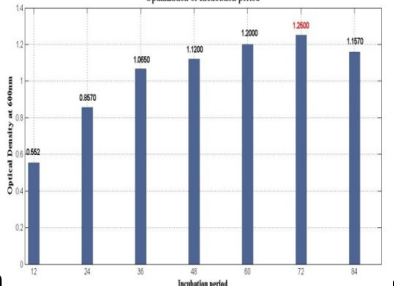

Figure 2. Optimization of $\mathrm{pH}$, Temperature an

eudomonas fluorescens

2C - Incubation period (Pseudomonas)

\section{Shelf life study for Bioinoculants}

Shelf life study of 120 days for Azospirillum lipoferum and Pseudomonas fluorescence in powder carrier's material is given in Figure 3-A, B. Among the three powder carriers used saw dust was proved to have highest cell count of $10^{9} \mathrm{cfu} / \mathrm{ml}$ for both the organisms.

\section{A) Azospirillum lipoferum}

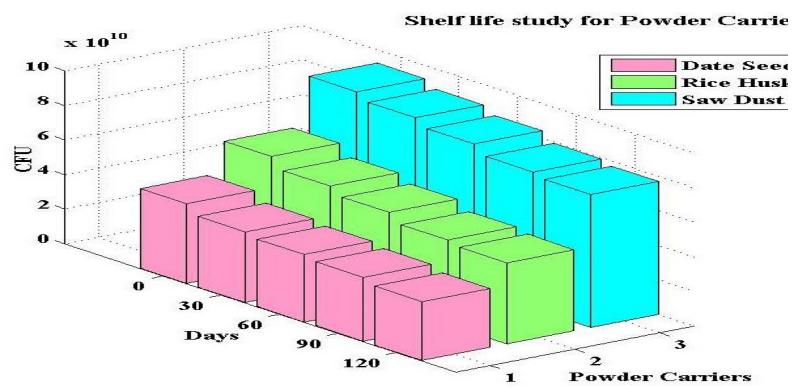

\section{B) Pseudomonas flouroscences}

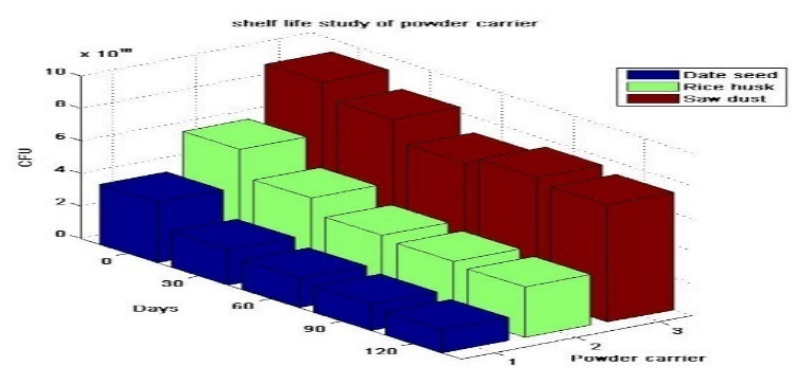

Figure 3. Shelf life study for powder carriers using MATLAB 
Gandhi et al., 2009 reported the effect of vermicompost in maintaining the shelf life of bioinoculant such as Azospirillum lipoferum, Bacillus megaterium and Pseudomonas fluorescens after 12 months in comparison with lignite carrier. Among Vermicompost based bioinoculants, B.megaterium showed maximum population of 7.60 x $10^{8} \mathrm{cfu} / \mathrm{g}$ dry weight on $360^{\text {th }}$ day followed by Pseudomonas $10^{8} \mathrm{cfu} / \mathrm{g}$ dry weight respectively.

\section{A) Azospirillum lipoferum}

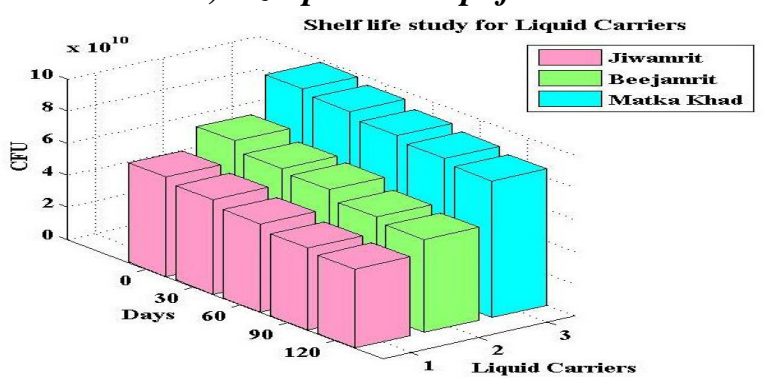

\section{Liquid Formulations}

Shelf life study of 120 days for Azospirillum lipoferum and Pseudomonas fluorescence in liquid carrier material is given in Figure 4-A, B. Among the three liquid carriers used Matka khad was proved to have highest cell count of $10^{9} \mathrm{cfu} / \mathrm{ml}$ for both the organisms.

Figure 4. Shelf life study for liquid carriers using MATLAB

Kavi et al., 2014 reported the survival of these three PGPR strains in liquid formulations amended with additives PVP, trehalose and glycerol for a period of six months storage. The results revealed that the required population $\left(1 \times 10^{8}\right.$ cells $/ \mathrm{ml}$ ) of saline tolerant strains was maintained both in carriers and in liquid based formulation.
Morphological characters for onion Powder carriers for Azospirillum lipoferum and Pseudomonas fluorescence (soil treatment).

Table 1.

\section{B) Pseudomonas flouroscences}

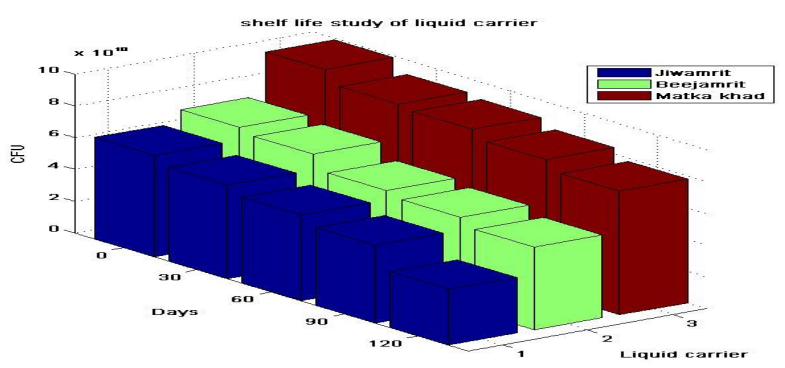

Table 1. Analysis of variance for powder carriers

\begin{tabular}{|c|c|c|c|c|c|c|c|c|c|c|c|c|}
\hline \multicolumn{13}{|c|}{ Azospirillum lipoferum $(S)+P$ seudomonas fluorescens $(S)$} \\
\hline \multicolumn{4}{|c|}{ Leaf length(cm) } & \multicolumn{3}{|c|}{ Leaf number } & \multicolumn{3}{|c|}{ Plant height $(\mathrm{cm})$} & \multicolumn{3}{|c|}{ Root length $(\mathrm{cm})$} \\
\hline $\begin{array}{l}\text { P.Cl } \\
\text { Vol.(g) }\end{array}$ & SD & RH & DSP & SD & RH & DSP & SD & RH & DSP & SD & RH & DSP \\
\hline C & 32.9000 & 32.9000 & 32.9000 & 7.0000 & 7.0000 & 7.0000 & 44.3000 & 44.3000 & 44.3000 & 9.0000 & 9.0000 & 9.0000 \\
\hline $\begin{array}{l}0.5(\mathrm{C} 1) \\
1.0(\mathrm{C} 2)\end{array}$ & $\begin{array}{l}28.1500 \\
27.5500\end{array}$ & $\begin{array}{l}32.1000 \\
35.0000\end{array}$ & $\begin{array}{l}25.1500 \\
33.2000\end{array}$ & $\begin{array}{l}7.5000 \\
10.0000\end{array}$ & $\begin{array}{l}12.5000 \\
12.5000\end{array}$ & $\begin{array}{l}9.5000 \\
13.5000\end{array}$ & $\begin{array}{l}51.4500 \\
51.5500\end{array}$ & $\begin{array}{l}51.5500 \\
55.7500\end{array}$ & $\begin{array}{l}42.3000 \\
52.6000\end{array}$ & $\begin{array}{l}21.1000 \\
21.4000\end{array}$ & $\begin{array}{l}17.2000 \\
18.3500\end{array}$ & $\begin{array}{l}15.1500 \\
17.1000\end{array}$ \\
\hline $1.5(\mathrm{C} 3)$ & 30.3500 & 36.0000 & 34.8000 & 11.5000 & 13.0000 & 14.0000 & 54.8833 & 57.5000 & 58.6000 & 22.1500 & 19.0000 & 21.2000 \\
\hline 2.0 (C4) & 30.6500 & 36.9500 & 34.9000 & 12.0000 & 14.0000 & 16.0000 & 56.4000 & 59.9500 & 59.3500 & 23.2500 & 20.5000 & 23.2000 \\
\hline & \multicolumn{3}{|c|}{$\begin{array}{l}\text { SED=2.15896 } \\
\text { CD(0.01) }=5.93714\end{array}$} & \multicolumn{3}{|c|}{$\begin{array}{l}\mathbf{S E D}=2.88675 \\
\mathbf{C D}(\mathbf{0 . 0 1})=7.93857\end{array}$} & \multicolumn{3}{|c|}{$\begin{array}{l}\text { SED= 5.34653 } \\
\text { CD }(\mathbf{0 . 0 1})=14.70295\end{array}$} & \multicolumn{3}{|c|}{$\begin{array}{l}\text { SED }=3.31937 \\
\text { CD }(\mathbf{0 . 0 1})=9.12827\end{array}$} \\
\hline
\end{tabular}

*Values are mean of the duplicate. P.C-Powder carriers, SD-Saw Dust, RH-Rice Husk, DSP-Date Seeds Powder; S-soil, C- Control.

\section{Leaf length}

Among the carriers, Rice husk has shown the best carrier treatment than saw dust and date seeds powder (Figure 5).

\section{Leaf number}

There is no significant difference among all the carriers and the concentration.

\section{Plant height}

Among the carriers, Rice husk has shown the best carrier treatment than saw dust and date seeds powder. C4 \&C5 of the entire carrier has shown best treatmentsthanC1, C2 \& C3.

\section{Root length}

There is no significant difference among all the carriers and the concentration (Table 2). 

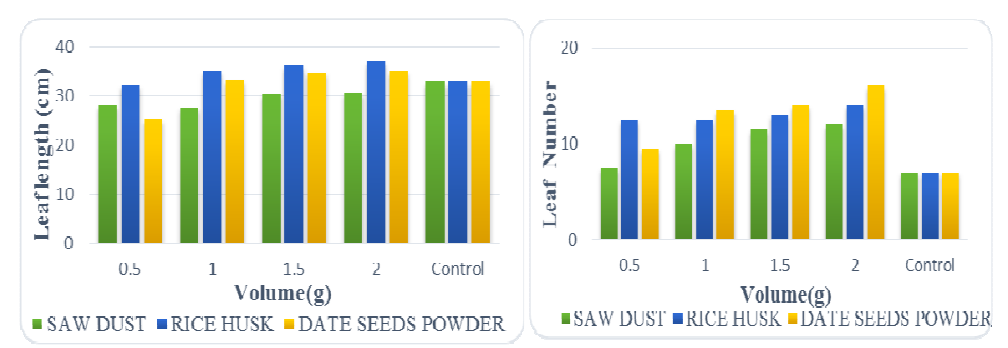

A) Leaf length

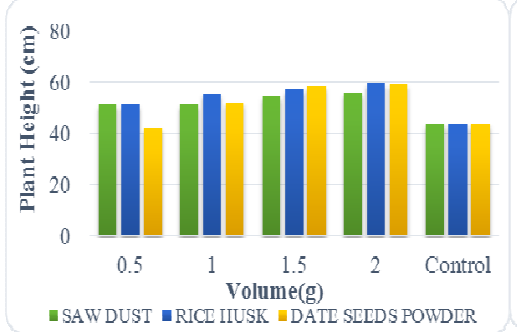

C) Plant height
B) Leaf number

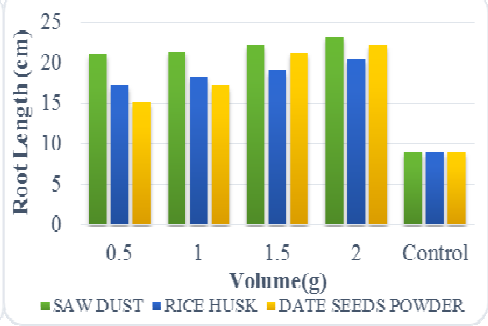

D) Root length

Figure 5. Plant growth parameters-A) Leaf length, B) Leaf number, C) Plant height \& D) Root length

Table 2. Analysis of variance for powder carriers

\begin{tabular}{|c|c|c|c|c|c|c|c|c|c|}
\hline \multicolumn{10}{|c|}{ Azospirillum lipoferum(S)+Pseudomonas fluorescens $(S)$} \\
\hline \multicolumn{4}{|c|}{ Bulb weight(g) } & \multicolumn{3}{|c|}{ Bulb diameter(cm) } & \multicolumn{3}{|c|}{ Neck diameter(cm) } \\
\hline P.C/Vol. (g). & SD & RH & DSP & SD & RH & DSP & SD & RH & DSP \\
\hline C & 2.2400 & 2.2400 & 2.2400 & 4.6000 & 4.6000 & 4.6000 & 3.0000 & 3.0000 & 3.0000 \\
\hline $0.5(\mathrm{C} 1)$ & 5.6133 & 4.7333 & 3.1533 & 6.7500 & 6.2000 & 5.8500 & 3.7500 & 3.7000 & 2.6000 \\
\hline 1.0 (C2) & 5.7733 & 6.5300 & 9.9000 & 6.1333 & 6.8500 & 7.7000 & 3.8000 & 4.2000 & 4.6000 \\
\hline 1.5 (C3) & 10.0033 & 6.2100 & 10.9433 & 9.4000 & 7.4500 & 7.0500 & 5.8000 & 4.8500 & 4.9000 \\
\hline 2.0 (C4) & 10.4400 & 7.7933 & 11.2033 & 9.7000 & 7.7500 & 7.5433 & 6.7500 & 5.0500 & 5.0000 \\
\hline & \multicolumn{3}{|c|}{$\begin{array}{l}\text { SED }=1.47656 \\
\mathbf{C D}(\mathbf{0 . 0 1})=4.06054\end{array}$} & \multicolumn{3}{|c|}{$\begin{array}{l}\text { SED }=0.84977 \\
\text { CD }(\mathbf{0 . 0 1})=2.33686\end{array}$} & \multicolumn{3}{|c|}{$\begin{array}{l}\text { SED }=0.83240 \\
\text { CD }(\mathbf{0 . 0 1})=2.28910\end{array}$} \\
\hline
\end{tabular}

*Values are mean of the duplicate. P.C-Powder carriers, SD-Saw Dust, RH-Rice Husk, DSP-Date Seeds Powder, S-soil, C-Control

\section{Bulb weight}

Among the carriers, Date seeds power has shown the best treatment than rice husk and saw dust.C4 \& C5 have shown the best treatment than control (Figure 6).

\section{Bulb diameter}

There is no significant difference among all the carriers. C4 \& C5 have shown best treatments than control.

\section{Neck diameter}

There is no significant difference among all the carriers. C4 \& C5 have shown best treatments than control. 


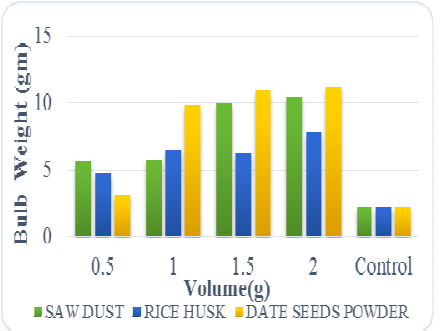

A) Bulb weight

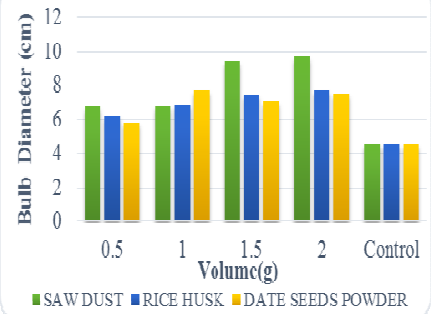

B) Bulb diameter

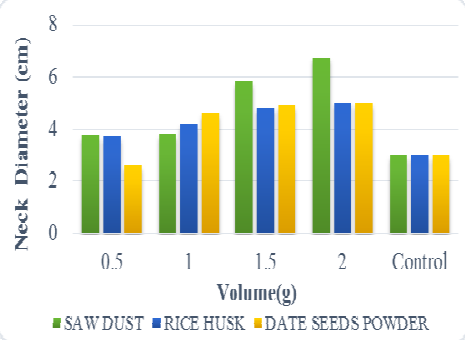

C) Neck diameter

Figure 6. Yield parameters are a) Bulb weight, b) Bulb diameter \& c) Neck diameter

Powder carriers for Azospirillum lipoferum and

Pseudomonas fluorescence (foliar spray

treatment)

The results are summarized in Table 3 .

Table 3. ANOVA of growth parameter for powder carrier

\begin{tabular}{|c|c|c|c|c|c|c|c|c|c|c|c|c|}
\hline \multicolumn{13}{|c|}{ Azospirillum lipoferum $(F S)+P$ seudomonas fluorescens $(F S)$} \\
\hline \multicolumn{4}{|c|}{ Leaf length(cm) } & \multicolumn{3}{|c|}{ Leaf number } & \multicolumn{3}{|c|}{ Plant height (cm) } & \multicolumn{3}{|c|}{ Root length(cm) } \\
\hline P.C./Vol & SD & RH & DSP & SD & RH & DSP & SD & RH & DSP & SD & RH & DSP \\
\hline \multicolumn{13}{|l|}{.(g) } \\
\hline $\mathbf{C}$ & 32.9000 & 32.9000 & 32.9000 & 7.0000 & 7.0000 & 7.0000 & 44.3000 & 44.3000 & 44.3000 & 9.0000 & 9.0000 & 9.0000 \\
\hline 0.5 (C1) & 28.8500 & 34.3167 & 25.1000 & 9.5000 & 9.5000 & 9.5000 & 49.7000 & 54.1000 & 44.1300 & 18.8000 & 20.2500 & 16.8800 \\
\hline $1.0(C 2)$ & 29.9500 & 33.1500 & 32.2500 & 10.5000 & 11.0000 & 14.0000 & 51.3000 & 56.3000 & 53.1700 & 18.9500 & 21.0500 & 18.4700 \\
\hline 1.5 (C3) & 30.3500 & 35.7500 & 35.0000 & 11.5000 & 13.5000 & 14.0000 & 54.7000 & 63.4000 & 58.1067 & 22.0500 & 25.0000 & 22.1733 \\
\hline $2.0(C 4)$ & 31.1833 & 38.6500 & 37.1000 & 14.0000 & 14.5000 & 16.0000 & 58.3500 & 67.8000 & 64.4500 & 23.4500 & 26.4500 & 24.0500 \\
\hline \multicolumn{4}{|c|}{$\mathbf{S E D}=2.83713$} & \multicolumn{3}{|c|}{ SED $=2.24846$} & \multicolumn{3}{|c|}{ SED $=3.90456$} & \multicolumn{3}{|c|}{ SED $=1.98247$} \\
\hline & \multicolumn{3}{|c|}{$\mathbf{C D}(\mathbf{0 . 0 1})=7.80210$} & \multicolumn{3}{|c|}{$\mathbf{C D}(\mathbf{0 . 0 1})=6.18325$} & \multicolumn{3}{|c|}{$\mathbf{C D}(\mathbf{0 . 0 1})=10.73754$} & \multicolumn{3}{|c|}{$\mathbf{C D}(\mathbf{0 . 0 1})=5.45180$} \\
\hline
\end{tabular}

*Values are mean of the duplicate SD - Saw dust, RH - Rice husk, DSP - Date seed powder, FS-Foliar spray, C-Control

\section{Leaf length}

After analysis it has been found that among the carriers saw dust has shown the best treatment. C4, C5 (1.5 gm, $2 \mathrm{gm})$ of saw dust have shown the best result and C2,C3 (0.5 gm, $1 \mathrm{gm})$ have shown poor result. Date seed powder have shown the poor treatment among the entire carrier. C2,C1 (0.5 gm and control) of Date seed and control of rice husk have shown the poor treatment (Figure 7).

\section{Leaf number}

While considering leaf number among the carrier Rice husk and date seed powder have shown the best treatment and Saw dust has shown the poor treatment.C4,C5 (1.5 gm, $2 \mathrm{gm})$ of all the carrier have shown best result and control has shown the poor treatment.

\section{Plant height}

Similar to leaf length, plant height also high in Saw dust compared to rice husk and date seed carriers. Control of Rice husk, Date seed and Saw dust and C2 (0.5 gm) of Saw dust have shown the poor result. 


\section{Root length}

While considering root length there is no significant difference among all the carriers.
But among the concentration, C4 and C5 (1.5 gm, 2 gm) of all carriers have shown the best result than that of control.
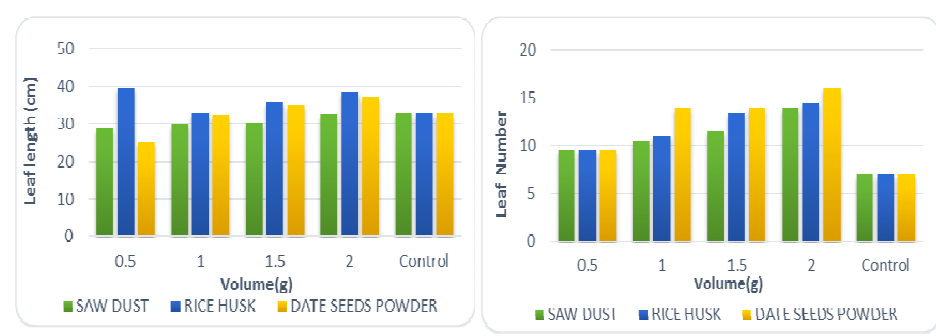

A) Leaf length

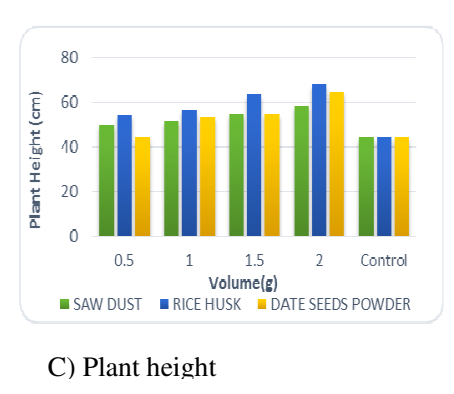

C) Plant height
B) Leaf number

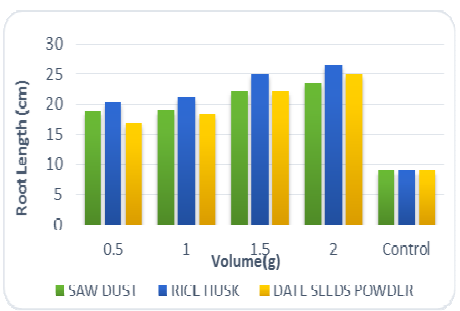

D) Root length

Figure 7- Plant growth parameters-A) Leaf length, B) Leaf number, C) Plant height \& D) Root length

\section{Bulb weight}

After analyzing it has found that among the carrier Saw dust and Date seed have shown the best result while Rice husk has shown the poor result. Among the volume compared to control, C3, C4 and C5 (1.0 gm, $1.5 \mathrm{gm}, 2 \mathrm{gm})$ have shown best treatment (Table 4 and Figure 8).

\section{Bulb diameter}

While considering bulb diameter there is no significant difference in the carrier but among the volume $\mathrm{C} 3, \mathrm{C} 4$ and $\mathrm{C} 5$ (1 gm, $1.5 \mathrm{gm}, 2 \mathrm{gm})$ have shown best result than that of $\mathrm{C} 2(0.5 \mathrm{gm})$ and control.

\section{Neck diameter}

Date seed powder and Rice husk have shown highest neck diameter compared to saw dust carrier. Among the volume C5 (2 gm) of all carriers shown better result while control and C2 $(0.5 \mathrm{gm})$ have reported to be poor treatment.

Table 4. ANOVA of growth parameter for powder carrier

\begin{tabular}{|c|c|c|c|c|c|c|c|c|c|}
\hline \multicolumn{10}{|c|}{ Azospirillum lipoferum $(F S)+P$ seudomonas fluorescens $(F S)$} \\
\hline & \multicolumn{3}{|c|}{ Bulb weight(g) } & \multicolumn{3}{|c|}{ Bulb diameter(cm) } & \multicolumn{3}{|c|}{ Neck diameter(cm) } \\
\hline $\begin{array}{l}\text { P.C / } \\
\text { Vol. (g). }\end{array}$ & SD & RH & DSP & SD & RH & DSP & SD & RH & DSP \\
\hline C & 2.2400 & 2.2400 & 2.2400 & 4.6000 & 4.6000 & 4.6000 & 3.0000 & 3.0000 & 3.0000 \\
\hline 0.5 (C1) & 4.1833 & 5.0633 & 4.8300 & 5.2500 & 7.4500 & 5.6500 & 4.0000 & 4.1000 & 2.8500 \\
\hline $1.0(\mathrm{C} 2)$ & 4.7300 & 6.3833 & 10.2433 & 5.3500 & 8.2000 & 8.0500 & 4.5500 & 4.0000 & 4.4500 \\
\hline 1.5 (C3) & 5.4300 & 7.2833 & 10.2400 & 6.4000 & 8.3000 & 8.2000 & 4.6000 & 4.4000 & 4.6000 \\
\hline 2.0 (C4) & 6.7500 & 8.2233 & 12.9700 & 6.6000 & 9.3500 & 9.1000 & 5.9000 & 5.3167 & 4.7000 \\
\hline & \multicolumn{3}{|c|}{$\begin{array}{l}\text { SED }=1.77646 \\
\text { CD }\end{array}$} & \multicolumn{3}{|c|}{$\begin{array}{l}\text { SED }=1.01336 \\
\mathbf{C D}(\mathbf{0 . 0 1})=2.78673\end{array}$} & \multicolumn{3}{|c|}{$\begin{array}{l}\text { SED }=0.96686 \\
\mathbf{C D}(\mathbf{0 . 0 1})=2.65886\end{array}$} \\
\hline
\end{tabular}

\footnotetext{
*Values are mean of the duplicate. P.C-Powder carriers, SD-Saw Dust, RH-Rice Husk, DSP-Date Seeds Powder, FS- Foliar Spray , CControl
} 


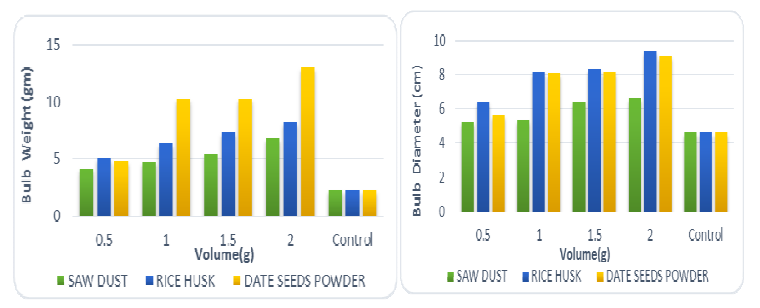

$\begin{array}{lll}\text { A) Bulb weight } & \text { B) Bulb diameter }\end{array}$

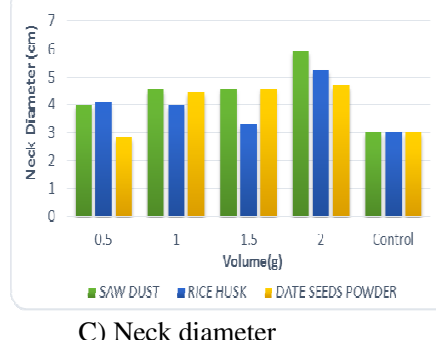

Figure 8. Yield parameters are a) Bulb weight, b) Bulb diameter \& c) Neck diameter

Liquid Carriers for Azospirillum lipoferum and Pseudomonas fluorescence (soil treatment)

Figure 9.

The results are presented in Table 5 and

\section{Leaf length}

Among the carriers, Rice husk has shown the best carrier treatment than saw dust and date seed powder.

\section{Leaf number}

There is no significant difference among all the carriers and the concentration.

\section{Plant height}

Among the carriers, Rice husk has shown the best carrier treatment than saw dust and date seed powder. C4 \& C5 of all the carrier have shown best treatments than $\mathrm{C} 1, \mathrm{C} 2 \& \mathrm{C} 3$.

\section{Root length}

There is no significant difference among all the carriers and the concentration.

Table 5. Analysis of variance for liquid carriers

\begin{tabular}{|c|c|c|c|c|c|c|c|c|c|c|c|c|}
\hline \multicolumn{13}{|c|}{ Azospirillum lipoferum $(S)+P s e u d o m o n a s$ fluorescens $(S)$} \\
\hline \multicolumn{4}{|c|}{ Leaf length(cm) } & \multicolumn{3}{|c|}{ Leaf number } & \multicolumn{3}{|c|}{ Plant height $(\mathbf{c m})$} & \multicolumn{3}{|c|}{ Root length(cm) } \\
\hline L.C./ & MK & BM & JM & MK & BM & JM & MK & BM & JM & MK & BM & JM \\
\hline \multicolumn{13}{|l|}{ Vol.(ml) } \\
\hline $\mathbf{C}$ & 31.8000 & 31.8000 & 31.8000 & 11.0000 & 11.0000 & 11.0000 & 50.9000 & 50.9000 & 50.9000 & 16.9000 & 16.9000 & 16.9000 \\
\hline 0.5 (C1) & 28.3500 & 32.4000 & 25.6500 & 12.5000 & 12.0000 & 7.5000 & 47.9500 & 51.4500 & 42.0500 & 17.3000 & 17.2000 & 14.5000 \\
\hline $1.0(\mathrm{C} 2)$ & 28.5500 & 34.4000 & 26.7500 & 13.0000 & 13.5000 & 11.0000 & 48.5167 & 57.7333 & 44.4000 & 17.1000 & 21.1000 & 15.5000 \\
\hline $1.5(\mathrm{C} 3)$ & 28.7000 & 37.1000 & 30.8500 & 15.5000 & 14.5000 & 12.0000 & 50.5500 & 62.6500 & 51.4500 & 19.9500 & 23.1500 & 18.3000 \\
\hline $2.0(\mathrm{C} 4)$ & 31.6500 & 37.3500 & 33.4000 & 10.0000 & 15.0000 & 14.0000 & 53.8500 & 64.8000 & 58.7667 & 20.2000 & 18.6167 & 23.3800 \\
\hline \multicolumn{4}{|c|}{ SED $=2.93096$} & \multicolumn{3}{|c|}{$\mathbf{S E D}=2.00278$} & \multicolumn{3}{|c|}{$\mathbf{S E D}=4.33611$} & \multicolumn{3}{|c|}{$\mathbf{S E D}=3.83832$} \\
\hline \multicolumn{4}{|c|}{$\mathbf{C D}(\mathbf{0 . 0 1})=8.06015$} & \multicolumn{3}{|c|}{$\mathbf{C D}(\mathbf{0 . 0 1})=5.50763$} & \multicolumn{3}{|c|}{$\mathbf{C D}(\mathbf{0 . 0 1})=11.92431$} & \multicolumn{3}{|c|}{$\mathbf{C D}(\mathbf{0 . 0 1})=10.55538$} \\
\hline
\end{tabular}

*Values are mean of the duplicate. L.C.-Liquid carriers, MK-Matka khad, BM-Beejamrit, JM-Jiwamrit, C-Control, S-Soil 


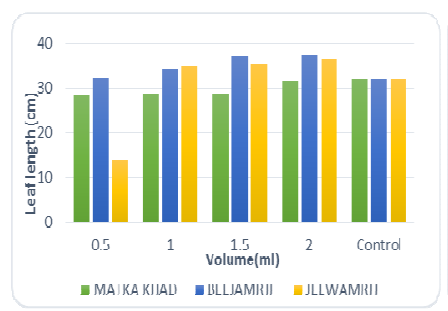

A) Leaf length

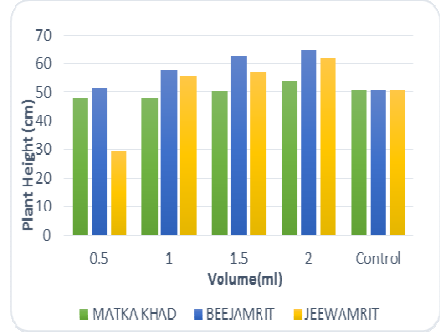

C) Plant height

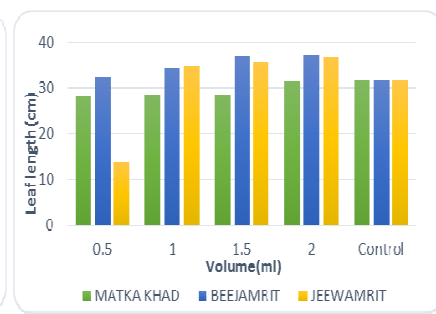

B) Leaf number

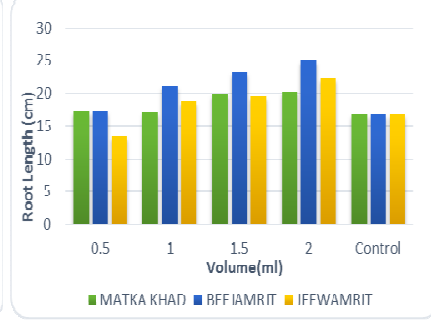

D) Root length

Figure 9. Plant growth parameters-A) leaf length, B) leaf number, C) plant height \& D) Root length

\section{Bulb weight}

Among the carriers, C2, C3, C4 \& C5 of saw dust and C4 \& C5 of Date seeds power have shown the best treatment.C2 \& C3of date seeds powder have shown the poor treatment (Table 6 and Figure 10).

\section{Bulb diameter}

$\mathrm{C} 1, \mathrm{C} 3, \mathrm{C} 4$ \& $\mathrm{C} 5$ of saw dust and Control of Date seeds power and rice husk have shown the best treatment. $\mathrm{C} 2$ of rice huskand $\mathrm{C} 2 \& \mathrm{C} 3$ of date seeds powder have shown the poor treatment.

\section{Neck diameter}

C4 \& C5 of saw dust have shown best treatments than $\mathrm{C} 2$ of date seeds powder.

Table 6. Analysis of variance for liquid carriers

\begin{tabular}{|c|c|c|c|c|c|c|c|c|c|}
\hline \multicolumn{10}{|c|}{ Azospirillum lipoferum $(S)+P$ seudomonas fluorescens $(S)$} \\
\hline \multicolumn{4}{|c|}{ Bulb weight(g) } & \multicolumn{3}{|c|}{ Bulb diameter(cm) } & \multicolumn{3}{|c|}{ Neck diameter(cm) } \\
\hline $\begin{array}{l}\text { L.C./Vol } \\
\text {.(ml) }\end{array}$ & MK & $\mathbf{B M}$ & $\mathbf{J M}$ & MK & $\mathbf{B M}$ & $\mathbf{J M}$ & MK & $\mathbf{B M}$ & JM \\
\hline C & 7.3500 & 7.3500 & 7.3500 & 8.5000 & 8.5000 & 8.5000 & 3.6000 & 3.6000 & 3.6000 \\
\hline 0.5 (C1) & 9.6733 & 4.7200 & 1.4500 & 7.3000 & 4.5333 & 3.3000 & 4.0500 & 2.7500 & 1.7000 \\
\hline 1.0 (C2) & 10.0300 & 5.3700 & 3.6833 & 7.4333 & 5.9500 & 4.4000 & 5.1500 & 3.2000 & 2.3500 \\
\hline 1.5 (C3) & 10.3733 & 6.2133 & 7.7300 & 7.7500 & 6.2500 & 7.0000 & 5.7000 & 3.3000 & 3.4000 \\
\hline $2.0(\mathrm{C} 4)$ & 10.4100 & 6.2300 & 8.1800 & 8.6000 & 6.3000 & 7.0500 & 6.0500 & 3.6000 & 3.7500 \\
\hline \multicolumn{4}{|c|}{$\begin{array}{l}\text { SED }=1.39437 \\
\text { CD }(\mathbf{0 . 0 1})=3.83450\end{array}$} & \multicolumn{3}{|c|}{ SED $=0.63584$} & \multicolumn{3}{|c|}{ SED $=0.17575$} \\
\hline
\end{tabular}

*Values are mean of the duplicate. P.C-Powder carriers, SD-Saw Dust, RH-Rice Husk, DSP-Date Seeds Powder,C-Control 


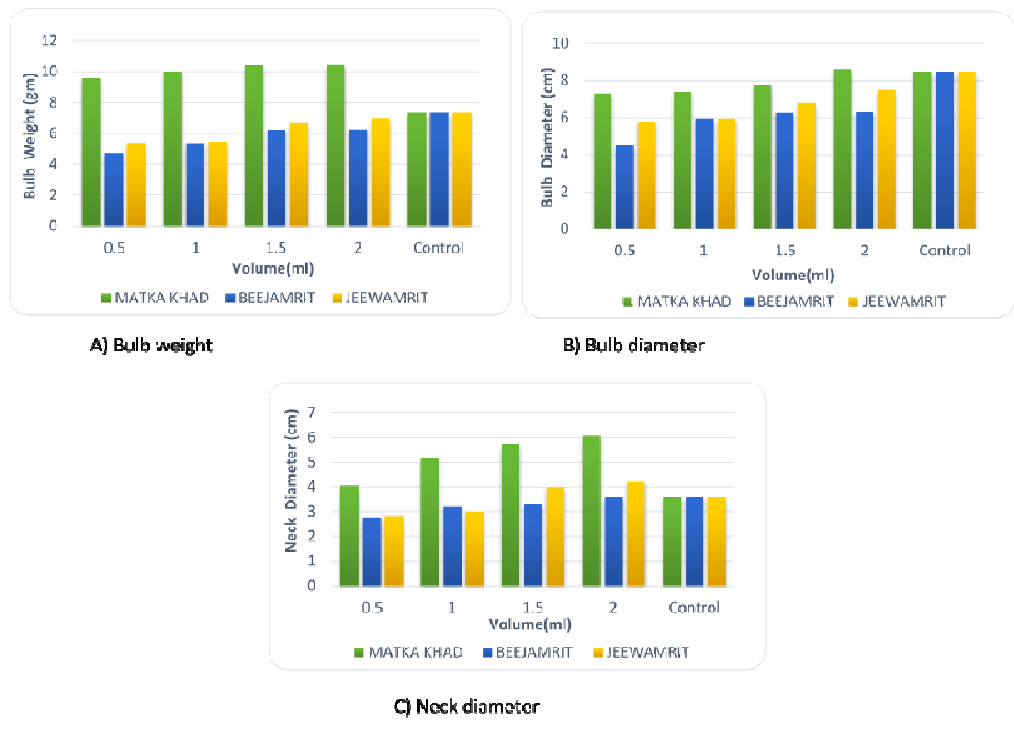

Figure 10. Yield parameters are a) Bulb weight, b) Bulb diameter \& c) Neck diameter

Liquid Carriers for Azospirillum lipoferum and Pseudomonas fluorescence (foliar spray treatment)

\section{Leaf length}

There is no significant difference among the different liquid formulation. All the carriers shown similar results in this parameter. But among the volume C3,C4,C5 $(1.0 \mathrm{ml}, 1.5 \mathrm{ml}, 2.0 \mathrm{ml})$ shows the best result and $\mathrm{C} 2(0.5 \mathrm{ml})$ have shown the poor result (Table 7 and Figure 11).

\section{Leaf number}

While considering this parameter, among the carrier Beejamrit has shown high significance and best result while Jeewamrit and Matkakhad have shown the poor result. Among the concentration $\mathrm{C} 2, \mathrm{C} 3$ that is $0.5 \mathrm{ml}$ and $1.0 \mathrm{ml}$ have shown poor treatment and $\mathrm{C} 4, \mathrm{C} 5(1.5 \mathrm{ml}, 2.0 \mathrm{ml})$ has reported to be the best treatment.

\section{Plant height}

According to the analysis of the data it has been found that there is no significant difference among the carrier but among the concentration $\mathrm{C} 4$, C5 $(1.0 \mathrm{ml}$, and $2.0 \mathrm{ml})$ have shown the best treatment and $\mathrm{C} 2(0.5 \mathrm{ml})$ has shown the poor treatment.

\section{Root length}

While considering root length, there is no significant difference among the carrier, but among the concentration $\mathrm{C} 1, \mathrm{C} 2, \mathrm{C} 3$ have (control, $0.5 \mathrm{ml}$, $1.0 \mathrm{ml}$ ) have shown poor treatment and $\mathrm{C} 4, \mathrm{C} 5$ $(1.5 \mathrm{ml}, 2.0 \mathrm{ml})$ has shown the best treatment.

Table 7. Analysis of variance for liquid carriers

\begin{tabular}{|c|c|c|c|c|c|c|c|c|c|c|c|c|}
\hline \multicolumn{13}{|c|}{ Azospirillum lipoferum $(F S)+$ Pseudomonas fluorescens $(F S)$} \\
\hline \multicolumn{4}{|c|}{ Leaf length $(\mathrm{cm})$} & \multicolumn{3}{|c|}{ Leaf number } & \multicolumn{3}{|c|}{ Plant height(cm) } & \multicolumn{3}{|c|}{ Root length(cm) } \\
\hline $\begin{array}{l}\text { L.C./ } \\
\text { Vol.(ml) }\end{array}$ & MK & $\mathbf{B M}$ & $\mathbf{J M}$ & MK & $\mathbf{B M}$ & $\mathbf{J M}$ & MK & $\mathbf{B M}$ & $\mathbf{J M}$ & MK & $\mathbf{B M}$ & JM \\
\hline C & 31.8000 & 31.8000 & 31.8000 & 11.0000 & 11.0000 & 11.0000 & 50.9000 & 50.9000 & 50.9000 & 16.9000 & 16.9000 & 16.9000 \\
\hline $0.5(\mathrm{C} 1)$ & 30.8500 & 30.8000 & 26.9333 & 7.0000 & 7.5000 & 10.0000 & 130.8500 & 45.7000 & 49.2000 & 17.4000 & 13.1000 & 20.5000 \\
\hline 1.0 (C2) & 31.0500 & 32.4000 & 29.2500 & 9.5000 & 13.0000 & 10.5000 & 31.0500 & 51.1000 & 54.4000 & 17.7000 & 16.4500 & 23.5500 \\
\hline $1.5(\mathrm{C} 3)$ & 31.9500 & 33.6500 & 29.4500 & 10.5000 & 13.0000 & 11.6667 & 31.9500 & 51.5500 & 55.6000 & 18.0500 & 17.8000 & 23.6500 \\
\hline $2.0(\mathrm{C} 4)$ & 33.1000 & 43.8500 & 35.1500 & 12.5000 & 20.0000 & 12.0000 & 33.1000 & 66.2000 & 66.1500 & 19.2667 & 19.9500 & 28.4500 \\
\hline $\begin{array}{l}\text { SED }=3.2 \\
\mathrm{CD}(0.01)=\end{array}$ & $\begin{array}{l}35 \\
3.91372 \\
\end{array}$ & & & $\begin{array}{l}\text { SED }=3 . \\
\text { CD(0.01) }\end{array}$ & $\begin{array}{l}972 \\
8.55174\end{array}$ & & $\begin{array}{l}\text { SED }=37 \\
\mathrm{CD}(0.01)=\end{array}$ & $\begin{array}{l}1621 \\
104.26957\end{array}$ & & $\begin{array}{l}\text { SED }=2 \\
\mathrm{CD}(0.01\end{array}$ & $\begin{array}{l}137 \\
6.60378 \\
\end{array}$ & \\
\hline
\end{tabular}

*Values are mean of the duplicate. L.C.-Liquid carriers, MK-Matka khad, BM-Beejamrit, JM-Jiwamrit, C-Control, FS-Foliar spray 


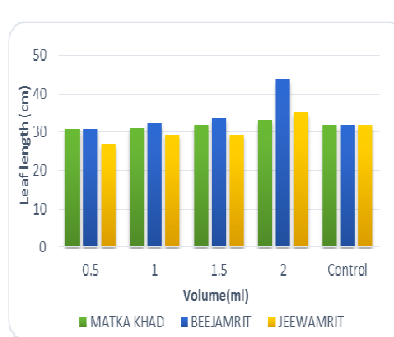

A) I eaf length

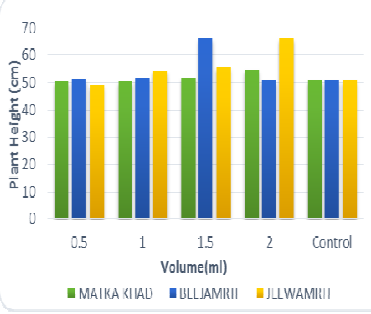

C) Plant height

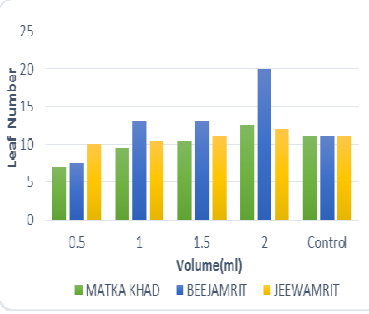

B) I eaf number

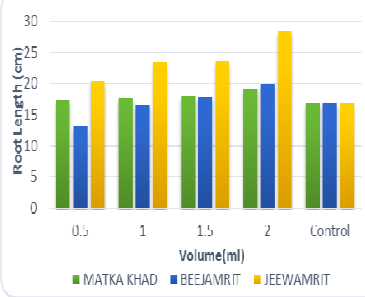

D) Root length

Figure 11. Plant growth parameters-A) leaf length, B) Leaf number, C) Plant height \& D) Root length

\section{Bulb weight}

Among the carrier Beejamrit has shown significant difference and C5 (2ml) of Beejamrit has shown the best result. $\mathrm{C} 2$ and $\mathrm{C} 3(0.5 \mathrm{ml}$, and 1.0 $\mathrm{ml})$ of Matka khad and $\mathrm{C} 2(0.5 \mathrm{ml})$ of jeewamrit has shown the poor result (Table 8 and Figure 12)

\section{Bulb diameter}

After the analysis it is found that among the carriers Beejamrit has shown good results. C3, C4 and $\mathrm{C} 5(1.0 \mathrm{ml}, 1.5 \mathrm{ml}, 2.0 \mathrm{ml})$ of Beejamrit have shown the best treatment and Matkakhad's C3 and $\mathrm{C} 2(1.0 \mathrm{ml}, 0.5 \mathrm{ml})$ have shown the poor result.

\section{Neck diameter}

While considering this parameter, among the carrier Matkakhad has shown the poor treatment but Jeewamrit and Beejamrit have shown the best treatment. Among the volume C4 and C5 $(1.5 \mathrm{ml}$, $2.0 \mathrm{ml}$ ) has shown good result and the $\mathrm{C} 2$ and $\mathrm{C} 3$ $(0.5 \mathrm{ml}, 1.0 \mathrm{ml})$ have shown poor result.

It is been reported that Pseudomonas fluorescens and other PGPRs induced a significant increase in root and shoot length, nodules, weight and even protein content in Mungbean plant (Dhanya et al., 2014, Heidari et al., 2014, Maiyappan et al., 2010 ).

It is also found that Strains of Pseudomonas putida and Pseudomonas fluorescens have increased root and shoot elongation in canola, lettuce, tomato and also yields in potato, radishes, rice, sugar beet, tomato, lettuce, apple, citrus, beans, ornamental plants, and wheat (Brahmaprakash et al.,2012).

Table 8. ANOVA of yield parameters for liquid carrier

\begin{tabular}{|c|c|c|c|c|c|c|c|c|c|}
\hline \multicolumn{10}{|c|}{ Azospirillum lipoferum $(F S)+P$ seudomonas fluorescens $(F S)$} \\
\hline \multicolumn{4}{|c|}{ Bulb weight $(\mathrm{g})$} & \multicolumn{3}{|c|}{ Bulb diameter(cm) } & \multicolumn{3}{|c|}{ Neck diameter(cm) } \\
\hline L.C./Vol.(ml) & MK & BM & JM & MK & BM & JM & MK & BM & JM \\
\hline C & 7.3500 & 7.3500 & 7.3500 & 8.5000 & 8.5000 & 8.5000 & 3.6000 & 3.6000 & 3.6000 \\
\hline 0.5 (C1) & 2.5400 & 4.1933 & 3.2133 & 3.6000 & 3.8500 & 4.4333 & 3.0000 & 3.0000 & 3.3000 \\
\hline 1.0 (C2) & 3.3100 & 8.9833 & 3.7833 & 5.9500 & 6.2500 & 5.9000 & 3.1500 & 4.3500 & 3.4333 \\
\hline 1.5 (C3) & 5.9200 & 9.2200 & 3.8400 & 6.7500 & 6.5500 & 5.9333 & 4.0000 & 4.5500 & 3.5000 \\
\hline 2.0 (C4) & 8.0267 & 12.1700 & 9.0833 & 7.3500 & 6.7000 & 7.1000 & 4.4833 & 4.9000 & 3.7500 \\
\hline \multicolumn{4}{|c|}{$\begin{array}{l}\text { SED= 2.00266 } \\
\mathbf{C D}(\mathbf{0 . 0 1})=5.50730\end{array}$} & \multicolumn{3}{|c|}{$\begin{array}{l}\text { SED }=0.91364 \\
\mathbf{C D}(0.01)=2.51251\end{array}$} & \multicolumn{3}{|c|}{$\begin{array}{l}\text { SED }=0.67245 \\
\mathbf{C D}(\mathbf{0 . 0 1})=1.84923\end{array}$} \\
\hline
\end{tabular}

*Values are mean of the duplicate. L.C.-Liquid carriers, MK-Matka khad, BM-Beejamrit, JM-Jiwamrit, C-Control, FS- Foliar spray 


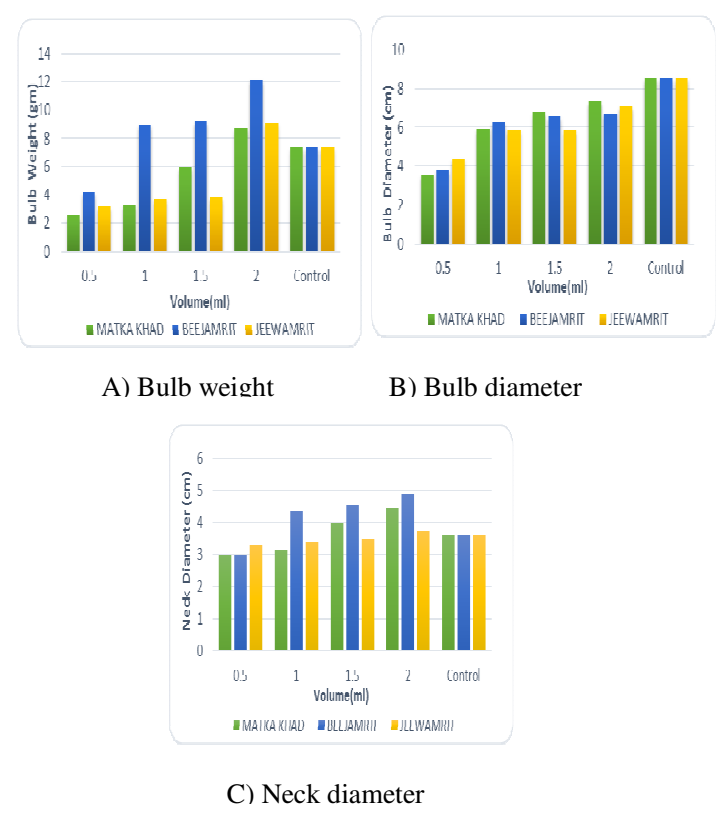

Figure 12. Analysis of yield parameter for liquid carrier

\section{CONCLUSIONS}

The highest cell concentration of $10^{9} \mathrm{CFU}$ was obtained for A.lipoferum at $\mathrm{pH} 7$, in $31^{\circ} \mathrm{C}$ after 74 hours of incubation and for Pseudomonas fluorescens maximum cell concentration is obtained at $\mathrm{pH} 8$, temperature $30^{\circ} \mathrm{C}$ and 72 hours of incubation respectively.

Different effective formulation of both powder and liquid carriers such as saw dust, rice husk, and date seed powder, Matka khad, Jiwamrit and Beejamrit respectively, were mixed with the optimised culture, which promotes the growth of the bacteria and produce an effective biofertilizer. Among the carriers (powder and liquid), highest cell viability was obtained in saw dust and Matka khad even after 120 days of storage.

For powder formulations, most of the carriers shown same result than control plant. Rice husk and date seeds powder have shown best result than saw dust.. Saw dust and Matka khad were the effective powder and liquid carrier formulations respectively with highest cell viability after 120 days of storage.

With respect to field performance such as yield and growth parameters, Saw dust and Beejamrit have proved be the best powder and liquid carriers. Thus biofertilizers has proven to be effective source than chemical fertilizers and was considered to be safe for practising agriculture naturally.

RESUMO: Biofertilizante é um grupo de microorganismos benéficos utilizados para melhorar a produtividade do solo através da fixação de azoto atmosférico ou por solubilização de fósforo no solo. Eles também estimulam o crescimento vegetal através de síntese de substâncias promotoras do crescimento. No presente estudo, Azospirillum lipoferum é cultivado em um meio de azul de bromotimol sem nitrogênio $(\mathrm{Nfb})$ e Pseudomonas fluorescens num meio de King's B. A condição de bioprocesso foi optimizada para ambas as culturas e descobriram que Pseudomonas fluorescens mostraram maior crescimento a $300^{\circ} \mathrm{C}$ em pH 8 após 72 horas de incubação, enquanto que Azospirillum lipoferum mostraram maior concentração de células a $310^{\circ} \mathrm{C}$ em pH 7 , com um período de incubação de 72 horas. A cultura optimizada é misturada com diferentes formulações de pó e veículo líquido tal como serragem, casca de arroz, pó de semente de tâmaras, Matka khad, Jiwamrit e Beejamrit respectivamente. O estudo do prazo de validade para 0, 30, 60, 90 e 120 dias por contagem celular e método de espalhamento em placa mostrou que o prazo de validade do biofertilizante produzido a partir do pó e veículos líquidos teve grande quantidade de população microbiana viável até 120 dias de armazenamento. Entre inoculantes biológicos de base biofertilizantes, a serragem mostrou população máxima de 77x109 CFU/ml para Azospirillum lipoferum e 72 x $109 \mathrm{CFU} / \mathrm{ml}$ para a estirpe Pseudomonas no $120^{\circ}$ dia e um veículo líquido Matka khad mostrou 85x109 CFU/ml para Azospirillum lipoferum e 78x109 CFU/ml para Pseudomonas fluorescens. 
PALAVRAS-CHAVE: Biofertilizantes. Azospirillumlipoferum. Pseudomonas fluorescens. Transportadores em pó e formulações líquidas

\section{REFERENCES}

ANBUSELVI, S.; JEYANTHI, R.; KARUNAKARAN, C. M. Antifungal activity of Pseudomonasfluorescens and its biopesticide effect on plant pathogens.National Journal of Chembiosis, v. 1, n. 1, p. 15-18,2010

DOBEREINER, J.History and new perspective of diazotrophs in association with non-leguminous plants.Symbiosis, $v .13$, p. 1-13, 1992

DOBEREINER, J.; DAY, J. M. Association symbiosis in tropical grasses: Characterization of microorganisms and Dinitrogen-fixing sites.Proceedings of the First International Symposium on Nitrogen Fixation, v. 2, p.518-538, 1976

DESHWAL, V. K.; SINGH, S. B.; CHUBEY, A.; KUMAR,P. Isolation and characterization of Pseudomonas strains from Potatoes Rhizosphere at Dehradun Valley India.International Journal of Basic and Applied sciences, v. 2, n. 2, p. 53-55, 2013

FEKADU, A. Isolation of Pseudomonas fluorescens from rhizospheric soil of faba bean and assessment of their Phosphate solubility: in vitro study Ethiopia. Scholars Academic Journal of Biosciences, v. 1, n. 7, p. 346351,2013

GANDHI, A.; SARAVANAKUMAR, K. Studies on shelf life of Azospirillum lipoferum, Bacillus megaterium and Pseudomonas fluorescens in Vermicompost carrier.Journal of Phytology, v. 1, n. 2, p. 100-107, 2009

HASARIN, N.; VIYADA, K.The Study of Shelf Life for Liquid Biofertilizer from Vegetable Waste. AU. J.T, v. 11, n. 4, p. 204-208, 2008

HOSSAIN, M. M. D.; JAHAN, I. Azospirillum as biofertilizer and Bangladesh perspective. Banat's Journal of Biotechnology, v. 11, p. 69-88, 2015

JAMBHULKAR, P. P.; SHARMA, P. Development of bioformulation and delivery system of Pseudomonas fluorescens against bacterial leaf blight of rice Xanthomonas oryzae pv. Oryzae. Journal of Environmental biology, v. 35 , p. 843-849,2014

JORJANI, M.; HEYDARI, A.; ZAMANIZADEH, H. R.; REZAEE, S .; NARAGHI, L. Development of Pseudomonas fluorescens and Bacillus coagulans based bioformulations using organic and inorganic carriers and evaluation of their influence on growth parameters of sugar beet. Journal of biopesticide, v. 4, n. 2, p. 180-185, 2011

KAUSHAL, K.; SHUKLA, U. N.; DHARMENDRA, K.; ANIL KUMAR, P; PRASAD, S. K. Bio-Fertilizers for Organic Agriculture. Popular Kheti, v. 1, pp. 91-96, 2013

KAVI KARUNYA, S.; REETHA, D. Survival of saline tolerant PGPR in different carriers and liquid formulations. International Journal Advanced Research Biological Science, v. 1, n. 2, p. 179-183, 2014

MARJAN, J.; HEYDARI, A.; ZAMANIZADEH, H. R.; REZAEE, S.; NARAGHI, L. Development of Pseudomonas fluorescens and Bacillus coagulans based bioformulations using organic and inorganic carriers and evaluation of their influence on growth parameters of sugar beet. Journal of Biopesticide, v. 4, n. 2, p. 180-185,2010 
MOTIUR, R. M.; MUBASSARA, S.; SIRAJUL, H.; ZAHED, U. M. K. Effect of some environmental factors on the growth of Azospirillum species isolated from saline soils of satkhira district Bangladesh. Bangladesh Journal Microbiological, v. 23, n. 2, p. 145-148,2006

PREMA, P.; SELVARANI, M. Microbial Siderophore as a Potent Biocontrol Agent for Plant Pathogens. International Journal of Science and Research. v. 2, n. 7, p. 521-523, 2013

RAMYASMRUTHI, S.;PALLAVI, O.; PALLAVI, S.; TILAK, K.; SRIVIDYA, S. Chitinolytic and secondary metabolite producing Pseudomonas fluorescensisolated from Solanaceaerhizosphere effective againstbroad spectrum fungal phytopathogens. Asian Journal of Plant Science and Research, v. 2, n. 1, p. 16-24, 2012.

RITIKA, B.; UTPAL, D. An overview of fungal and bacterial biopesticide to control plant pathogen/disease. African journal of microbiology research, v. 8, n. 17, p. 1749-1762, 2014

SANJAY, C.; RAMESHWAR; ASHLESHA.; SAINI, J. P.; PAUL, Y. S. Vedic krishi: sustainable live hood option for small and marginal farmers. Indian Journal of Traditional Knowledge, v. 11, n. 3, p. 480-486, 2012

SIVASAKTHIVELAN, P.; SARANRAJ, P. ‘Azospirillum and its formulations’, International Journal Microbiological Research, v. 4, n. 3, p. 275-287, 2013

SIVASAKTHIVELAN, P.; STELLA, D. Studies on the Efficacy of Different Formulations of Bioinoculants Consortium on Sunflower (Helianthus annuus.) Var. Modern. International Journal Current Advanced Research, v. 1, n. 2, p. $22-25,2012$

SOHEIL, S. A.; ASGHAR, H.; NEMAT ALLAH, K.R.A.; MAJID, E. Preparation of new biofungicides using antagonistic bacteria and mineral compounds for controlling cotton seedling damping-off disease. Journal of Plant Protection Research, v. 49, n. 1, p. 49-55, 2009

VIJENDRAKUMAR, R. C.; SREERAMU, B. S.; SHANKARAPPA, T. H.; SANTHOSH, K. V.; MALLIKARJUNAGOWDA, A. P.; UMESHA K. Effect of liquid bio fertilizers on growth, yield and Survival of seedlings in garden rue (Rutagraveolens Linn.). Plant Archives, v. 14, n. 1, p. 171-175, 2014

WAHYUDI, A. T.; RIKA, I. A.; GIYANTO. Screening of Pseudomonas Sp. Isolated from Rhizosphere of Soybean Plant as Plant Growth Promoter and Biocontrol Agent. American Journal of Agricultural and Biological Sciences, v. 6, n. 1, p. 134-141, 2011 